\title{
Agreement of clinical tests for the diagnosis of peripheral arterial disease
}

\section{Azzopardi Midolo Y, ${ }^{1}$ Gatt $A^{1,2}$, Chockalingam N ${ }^{2,1}$, Formosa C ${ }^{1,2}$}

${ }^{1}$ Faculty of Health Sciences, University of Malta

${ }^{2}$ School of Life Sciences and Education, Staffordshire University

*Corresponding Author: Dr Cynthia Formosa, PhD

Address for correspondence:

Room 14, Faculty of Health Sciences,

University of Malta,

Tal-Qroqq,

Msida, MSD 2080

Malta.

Fax: 23402342

Tel: 0035699861396

E-mail: Cynthia.formosa@um.edu.mt

Dr Alfred Gatt: Alfred.gatt@um.edu.mt

Prof Nachiappan Chockalingam: N.Chockalingam@staffs.ac.uk

Ms Yvonne Azzopardi Midolo: Yvonne.midolo@gmail.com 


\section{Abstract}

Aim: The aim of this study was to compare different screening modalities in the detection of PAD in a primary care setting.

Methods: Fifty participants living with Type 2 diabetes were recruited. Pulse Palpation, waveform analysis, ankle brachial pressure index, absolute toe pressure, toe brachial pressure index and transcutaneous oxygen pressure were compared in the detection of peripheral arterial disease. One hundred limbs were included for analysis.

Results: This study showed different results in peripheral arterial disease screening tests in the same group of participants. The highest percentage of participants who had PAD was for the Doppler Waveform (93.0\%). This was followed by TBPI (72.0\%), ABPI (57.0\%), ATP (35.0\%), TCPO (30.0\%) and Pulse Palpation (23.0\%). The difference between these percentages is significant $(p<0.0005)$. The magnitude of the effect size is medium/moderate (Cramer's V=0.498).

Conclusion: This study demonstrates that inconsistencies exist between the agreement of the 6 different modalities used to detect PAD. These findings should create an awareness amongst clinicians when interpreting results of these tests. The authors advocate for urgent, more robust studies utilizing a gold standard modality for the diagnosis of PAD in order to provide evidence regarding which screening modalities would yield the most valid results.

Keywords - peripheral arterial disease, diabetic foot, ankle brachial pressure index, spectral waveforms, pulse palpation, toe pressures, misdiagnosis 


\subsection{Introduction}

Diabetic foot disease is amongst one of the most feared complications of diabetes [1]. It is of global concern, being the leading cause of lower limb amputations [2] which results in reduced quality of life, and hospitalization, incurring a considerable financial burden [2].

Peripheral Arterial Disease (PAD) refers to the formation of atherosclerotic plaques in the blood vessels of the lower limbs. This results in reduced blood flow to the effected limbs and is a major risk factor for chronic foot ulcers and for lower limb amputations especially in individuals living with diabetes [3]. Patients with diabetes have a 2 - 3-fold increased risk of developing atherosclerosis $[1,4]$. For every $1 \%$ increase in HBA1c there is a corresponding $26 \%$ increased risk of developing PAD [5]. For these reasons, early diagnosis of PAD in people living with diabetes is imperative [6].

The real prevalence of PAD in patients with diabetes has been proven difficult to determine since most patients are asymptomatic [7] and approximately one half of the individuals living with PAD, remain undiagnosed. This is a result of two main factors; either because the individual is asymptomatic or has atypical symptoms or else because the health care professionals make use of poorly-validated methods of screening for PAD, such as pulse palpation or history of intermittent claudication. Most importantly, literature presents issues $[2,6]$ with regards to the validity of the current screening techniques commonly used in assessing lower limb perfusion, such as pulse palpation, which is subjective, calculation of ABPI and TBPI where their results is debateable due to the influence of peripheral neuropathy and arterial calcification and the accuracy of waveform analysis alone, since although reported as having high inter-rater reliability, the validity of this test remains unclear. 
Broad interest exists in improving the quality of primary health care, not only among clinicians and patients but also among politicians and insurers, therefore, since early correct diagnosis of PAD is crucial in the diabetic population [6], especially amongst asymptomatic patients presenting at primary care to prevent diabetic foot complications and lower limb amputations [8]. Literature suggests the need to correctly optimize the diagnosis of PAD especially in nonspecialist settings such as primary care [9] where there could be the possibility that clinicians differ in their screening and diagnostic skills when compared to a vascular specialist. Effective long term care of patients with PAD requires an increase in diagnostic efforts within the clinical setting to decrease ischemic complications, systemic cardiovascular risk and improve quality of life [10].

In 2011, Stephens et al. reported that the general practice for detecting PAD was mainly focused on pulse palpation, physical examination, history taking and patient reporting symptoms of intermittent claudication, the same methods of assessment for PAD which were used back in 1996 [11]. Although there is vast knowledge with regards to PAD, there seems to be lack of knowledge and consensus regarding the best method of assessment of PAD in patients living with diabetes $[12,13]$.

This gap in knowledge and the need for identifying the most appropriate method to accurately detect PAD has prompted the need to conduct this study. This research sought to compare 6 most commonly used clinical tools for diagnosis of PAD in the lower limbs, i.e. foot pulses, ankle brachial pressure index, toe brachial pressure index, spectral doppler waveform analysis, absolute toe pressure and transcutaneous oxygen perfusion $\left(\mathrm{TcP}_{2}\right)$, to look for agreement between them making this study the first to directly compare these 6 different modalities. 


\subsection{Materials and Methods}

A prospective non-experimental comparative study was conducted. Fifty participants living with Type 2 diabetes were recruited using a convenience sampling method. This study was approved by the University Research Ethics Committee and all participants provided informed consent before any data collection. All investigations were carried out in accordance with the principles of the Declaration of Helsinki as revised in 2000. Adults diagnosed with type 2 diabetes mellitus were recruited. Participants were excluded if they presented with open wounds which contraindicate compression and which could interfere with the assessment, foot/lower limb oedema, lower extremity amputation, history of revascularisation procedures of the lower limbs, conditions which contradict compression, a history of DVT in the previous 6 months, not being cognitively alert or showing unwillingness to participate in the study.

The testing modalities and examination methods were carried out by same investigator to ensure inter-rate reliability. The screening process involved review of the patient's medical history and a lower-extremity physical examination. Each individual's personal lifestyle characteristics and clinical history including duration of diabetes, recent $\mathrm{HbA1c}$, weight, height, current medications, smoking history were recorded. History of hypertension, hyperlipedaemia, ischemic related changes to the foot such as hair loss; atrophic, shiny skin were also noted.

All participants were assessed for PAD using the 6 different modalities described below, in random order. The right and left foot were assessed in each participant, therefore 100 limbs were included for analysis. Room temperature was maintained at 21 to 23 degrees Celsius (68 to $75^{\circ} \mathrm{F}$ ) during the assessments to avoid vasoconstriction of digital arteries from the cold. Participants were rested in the supine position and acclimatized for 20 minutes prior to testing. 


\subsection{Pedal Pulse Palpation}

Palpation of the foot pulses; namely the dorsalis pedis; which is located on the dorsal aspect of the foot between the first and second metatarsals; and the posterior tibial; which is located behind the medial malleolus were assessed. This assessment was conducted by an experienced vascular clinician. The feet were also examined to check for any changes such as: colour, pallor, cyanosis; extreme change in temperature gradient (colder distally); hair anomalies and atrophic, shiny, dry skin. Pulses were classified as present or absent. Absent pulses denoted presence of arterial disease.

\subsection{Spectral Doppler Waveform Analysis and ABPI Measurements}

Measurement of ABPI was performed using a portable hand held Doppler and blood pressure cuffs. Pedal spectral waveform analysis of the dorsalis pedis and the posterior tibial were acquired from all recruited subjects utilizing a Huntleigh® Dopplex Assist vascular package (Cardiff, UK) with an $8 \mathrm{MHz}$ probe. The probe was held steadily on the anatomical artery location at an angle between 45 to 60 degrees against the flow of arterial blood. Interpretation of arterial pressure waveforms results was based on standards from the literature [5]. Waveforms were classified as triphasic, biphasic, monophasic discontinuous and monophasic continuous. The triphasic waveforms were considered as normal, whereas the biphasic and monophasic discontinuous and monophasic continuous waveforms were interpreted as abnormal.

Participants received instructions refrain from smoking and caffeine intake prior to data collection. Measurements were carried out after a 5-minute rest in supine position with the upper body as flat as possible, with all tight clothing around the waist and the arm undone.

A blood pressure cuff was applied to the arm [to measure the brachial systolic pressure] and the ankle [to measure the dorsalis pedis and posterior tibial pressures] to determine the ankle 
pressure. The systolic pressure was noted and the higher values of the brachial and the ankle pressures were used to calculate the ABPI. Values were interpreted according to the criteria proposed by the American Heart Association and the American Diabetes Association [14]. ABPI calculations were interpreted as 0.9-1.29 normal, lower-extremity vascular disease was defined an ankle brachial index $<0.90$ in either foot. An ABPI of $>1.3$ was considered significantly elevated and indicative of vascular calcification.

\subsection{Toe Pressures - ATP and TBI}

The procedure for obtaining toe pressures and TBI's involved applying a $1.5 \mathrm{~cm}$ cuff on the proximal aspect of the hallux or $2^{\text {nd }}$ toe and the photopletysmography probe was applied on the pulp of the hallux. Patients were examined in the supine position. When a regular waveform was observed, the sphygmomanometer was pumped up slowly to inflate the digital cuff and occlude digital blood flow, to a maximum of $200 \mathrm{mmHg}$. Upon slow release, the point at which the waveform was observed the toe systolic pressure was measured. Using PPG, the actual TBI was obtained during gradual deflation of the cuff at the moment the pulsatile signal reappeared. Toe pressures and the TBI were automatically calculated by the dopplex unit.

For ATP results ranging from $75 \mathrm{mmHg}-130 \mathrm{mmHg}$ were considered as not having PAD, whilst any results $=<74 \mathrm{mmHg}$ were considered as an indication of periphera arterial disease (Kapur 2015). Participants with a TBPI ratio $>0.7$ were diagnosed as not having PAD, whilst those with a ratio of $<0.7$ were diagnosed with PAD (weinberg, 2012)

\section{$2.4 \mathrm{TcPO}_{2}$}

The $\mathrm{TcPO}_{2}$ measurement was carried out with the use of a Perimed PeriFlux 6000 machine (Perimed , 2017). The electrode, placed on the dorsal, tibial aspect of the midfoot, was 
calibrated automatically by the machine itself. Prior to calibration, the site of assessment was cleaned with alcohol after the removal of any hair growth.

A standard fixation ring was applied securely to the prepared site. The hole of the fixation ring was filled with contact liquid and the electrode was fixed into the ring.

Following the electrode fixation, a waiting time of 15 - 20 minutes was allowed for physiological stabilization. During this time, the electrode slowly heated the skin to vasodilate the arteries which is necessary to do the $\mathrm{tcpO}_{2}$ reading. Following the physiological stabilisation, the machine continued to perform the $\mathrm{tcpO}_{2}$ readings automatically.

Participants with a $\mathrm{TcPO}_{2}$ reading $=1>50 \mathrm{mmHg}$ were classified as having no $\mathrm{PAD}$, whilst those with a reading below this value were diagnosed as having PAD (REF).

For the purposes of data analysis, each foot was scored separately resulting in 100 limbs for analysis. All data was recorded on a spreadsheet designed in Microsoft Excel to group together the information required for interpretation of the results. The IBM SPSS (Statistical Package for Social Sciences) (Version 25) program was utilized for the statistical analysis. The Chi Square test was used to assess the association between two categorical variables. One of these variables was peripheral arterial disease (Present versus Absent) while the other variable included the instruments used (Pulse Palpation, waveform analysis, ankle brachial pressure index, absolute toe pressure and transcutaneous oxygen pressure). The null hypothesis specified that there is no association between the two categorical variables and was accepted if the p-value exceed the 0.05 level of significance. 


\subsection{Results}

A total of 50 participants with type 2 diabetes, 14 males and 36 females, mean age of 71.1 years, mean duration of diabetes, 18.82 years and mean weight $79.94 \mathrm{~kg}$, were included in the study.

The highest percentage of participants that were positive for PAD was reported by the Doppler Waveform (93.0\%). This was followed by TBPI (72.0\%), ABPI (57.0\%), ATP (35.0\%), TCPO2 (30.0\%) and Pulse Palpation (23.0\%). On the other hand, the highest proportion of respondents that did not have PAD was for the Pulse Palpation (77.0\%), followed by $\mathrm{TcPO}_{2}$ (70.0\%), ATP (65.0\%), ABPI (43.0\%), TBPI (28.0\%) and Doppler Waveform (7.05). The difference between these percentages is significant $(p<0.0005)$.

The Cramer's V is a test that can be used to yield an estimation of the magnitude of the association between the variables. Therefore, this test was used to determine the strength of the association between the 6 modalities for the assessment of PAD used in this study. According to Cramer's V , the strength (or magnitude) of association between the two variables is moderate (Cramer's $\mathrm{V}=0.498)$.

Insert Table 1 and Figure 1

\subsection{Discussion}

This study sought to compare the agreement of commonly-used non-invasive clinical tests utilized for the detection of PAD including pulse palpation, waveform analysis, ankle brachial pressure index, absolute toe pressure, toe brachial pressure index and transcutaneous oxygen pressure in subjects living with Type 2 diabetes mellitus. 
There is an extensive amount of literature available concerning various screening methods for $\mathrm{PAD}$, however to date there is no definite evidence on whether in fact, the results of these tests concur with each other. This proves to be a major concern since PAD is considered as one of the most dangerous complication of diabetes that increases the likelihood of ulceration and amputation.

Current study, whilst seeking to provide this required evidence, clearly demonstrates substantial inconsistencies between these 6 different screening/diagnostic modalities for PAD. One might argue that the major limitation of this study is that the 6 test results were not compared to a 'gold standard' test for PAD such as conventional angiogram or CT Angio, however this is the first study which has quantitatively compared all the presently available clinical tests for arterial perfusion. Results from pulse palpation reports the least presence of PAD (23\%) and doppler waveform the highest (93\%). Although one could argue that we are unable to determine the actual incidence of PAD in the study population since they were not investigated utilizing a gold reference as highlighted above, the main purpose of this paper is to compare commonly used clinical measurement techniques. These are the techniques used in everyday clinics around the world to make clinical decisions and to develop clinical management plans.

The findings reported within this paper have important implications for both clinical practice and future research. The reported observations suggest that use of only one screening tool in isolation, could yield high false results since it is clear that these tests do not concur with each other to a large extent. Although the use of more specialized investigations such as duplex scanning could be compared with the six modalities to detect PAD that this study focused on, it was beyond the scope of the reported investigation. This was mainly attributed to the frontline 
assessment carried out at the primary care level, with the main criteria being easier availability to the general medical practitioner. A future study could build on our results to identify the right modality which will be beneficial for the community.

The authors postulate that one possible reason for the increase of both minor and major amputations worldwide could be the untimely and/or incorrectly diagnosed PAD due to inconsistency exhibited between these 6 widely used tests. Patients who are falsely identified as having no PAD when indeed this could be present could pose a threat to this high risk population since if they are not appropriately detected, they would be denied early beneficial and effective secondary risk factor control together with further investigations to determine the extent of the condition. Furthermore, accurate diagnosis also safely reduces unnecessary secondary care referrals when it is known that these appointments could be utilized by those patients who truly have the condition and are denied of prompt attention due to long waiting lists.

We recommend that those practitioners who are clinically responsible for patients should be made aware of these inconsistencies, and possibly advised to use alternative methods of diagnosis, such as more detailed clinical evaluation and/or imaging modalities. Findings from this study have created an urgent need for replicating this study utilizing a reference standard modality for the diagnosis of PAD in order to provide sufficient evidence as to which tool should be utilized for the screening and diagnosis of this common condition which is often managed by the clinician in a primary care or general practice setting.

\subsection{Conclusion}


Correct diagnosis of PAD in patients with diabetes is crucial. This study demonstrates that inconsistencies exist between the agreement of the 6 different modalities used to detect PAD.

These findings should create an awareness amongst clinicians and researchers in the field, with regards to caution when interpreting results of these tests. The authors advocate for urgent, more robust studies utilizing a gold standard modality for the diagnosis of PAD in order to provide evidence regarding which non-invasive screening modalities would yield the most valid results. This would significantly reduce the proportion of patients with diabetes who would be falsely identified as having no PAD and subsequently denied beneficial and effective secondary risk factor control.

\section{Acknowledgements}

The authors would like to thank all participants who consented to participate in this study.

\section{Declaration of interest}

The authors report no conflict of interest. 


\section{References}

[1] Khanolkar MP, Bain SC, Stephens JW. The diabetic foot. QJM 2008;101:685-95. doi:10.1093/qjmed/hcn027.

[2] Williams DT, Harding KG, Price P. An evaluation of the efficacy of methods used in screening for lower-limb arterial disease in diabetes. Diabetes Care 2005;28:2206-10.

[3] Jude EB, Oyibo SO, Chalmers N, Boulton AJ. Peripheral arterial disease in diabetic and nondiabetic patients: a comparison of severity and outcome. Diabetes Care 2001;24:1433-7.

[4] Marso SP, Hiatt WR. Peripheral Arterial Disease in Patients With Diabetes. J Am Coll Cardiol 2006;47:921-9. doi:10.1016/j.jacc.2005.09.065.

[5] Norgren L, Hiatt WR, Dormandy JA, Nehler MR, Harris KA, Fowkes FG. Inter-Society Consensus for the Management of Peripheral Arterial Disease (TASC II). J Vasc Surg 2007;45. doi:10.1016/j.jvs.2006.12.037.

[6] Formosa C, Cassar K, Gatt A, Mizzi A, Mizzi S, Camileri KP, et al. Hidden dangers revealed by misdiagnosed peripheral arterial disease using ABPI measurement. Diabetes Res Clin Pract 2013;102:112-6. doi:10.1016/j.diabres.2013.10.006.

[7] Thiruvoipati T, Kielhorn CE, Armstrong EJ. Peripheral artery disease in patients with diabetes: Epidemiology, mechanisms, and outcomes. World J Diabetes 2015;6:961-9. doi:10.4239/wjd.v6.i7.961.

[8] Formosa C, Gatt A, Chockalingam N. Diabetic foot complications in Malta: prevalence of risk factors. Foot (Edinb) 2012;22:294-7. doi:10.1016/j.foot.2012.08.008.

[9] Lewis JE, Williams P, Davies JH. Non-invasive assessment of peripheral arterial disease: Automated ankle brachial index measurement and pulse volume analysis compared to duplex scan. SAGE Open Med 2016;4:2050312116659088. doi:10.1177/2050312116659088.

[10] Hirsch AT, Criqui MH, Treat-Jacobson D, Regensteiner JG, Creager MA, Olin JW, et al. Peripheral Arterial Disease Detection, Awareness, and Treatment in Primary Care. JAMA 2001;286:1317. doi:10.1001/jama.286.11.1317.

[11] Stephens J, Hagler D, Clark E. Got PAD? Hidden dangers revealed with ABI. J Vasc Nurs 2011;29:153-7. doi:10.1016/j.jvn.2011.08.002.

[12] Ikem R, Ikem I, Adebayo O, Soyoye D. An assessment of peripheral vascular disease in patients with diabetic foot ulcer. Foot 2010;20:114-7. doi:10.1016/j.foot.2010.09.002.

[13] Formosa C, Gatt A, Chockalingam N. A Critical Evaluation of Existing Diabetic Foot Screening Guidelines. Rev Diabet Stud 2016;13:158-86. doi:10.1900/RDS.2016.13.158. 
[14] Orchard T, Strandness D. Assessment of Peripheral Vascular Disease in Diabetes: Report and Recommendations of an International Workshop Sponsored by the American Heart Association and the American Diabetes Association. Diabetes Care 1993;16:1199-209. 


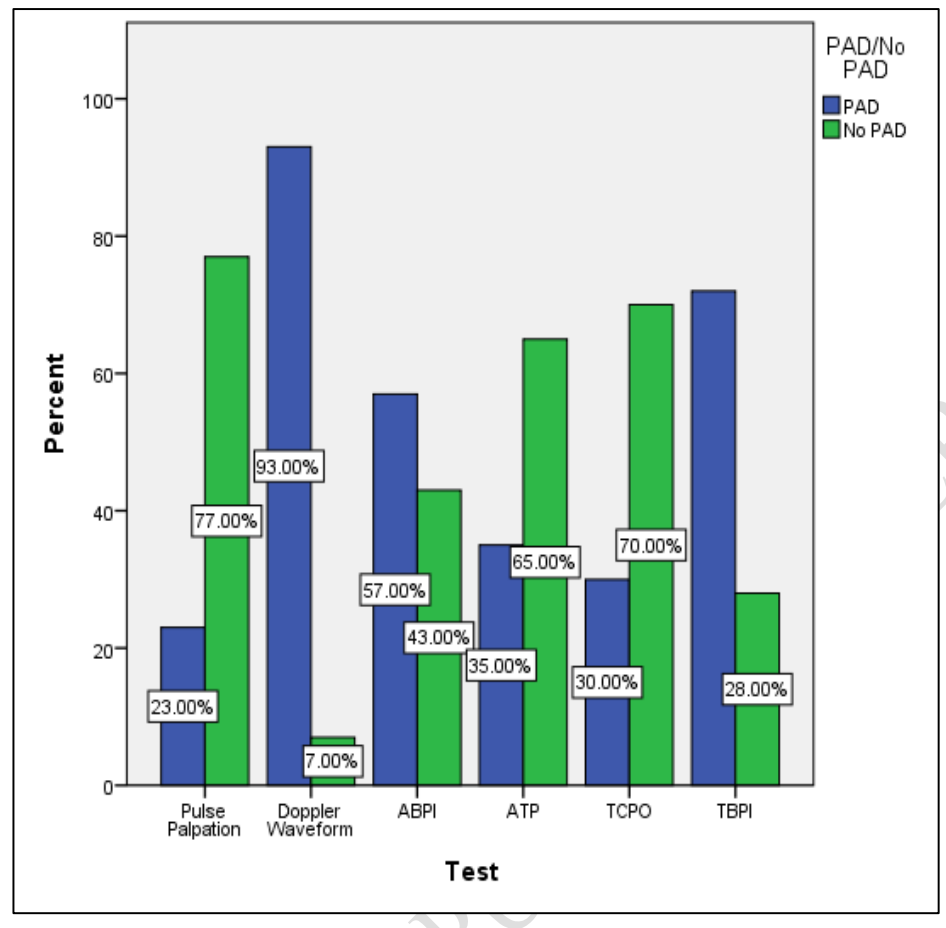

Figure 1: Presence/absence of PAD using the different physiological tests ( $\mathrm{n}=100$ limbs) $X^{2}(5)=148.939, p<0.0005$, Cramer's $V=0.498$ 


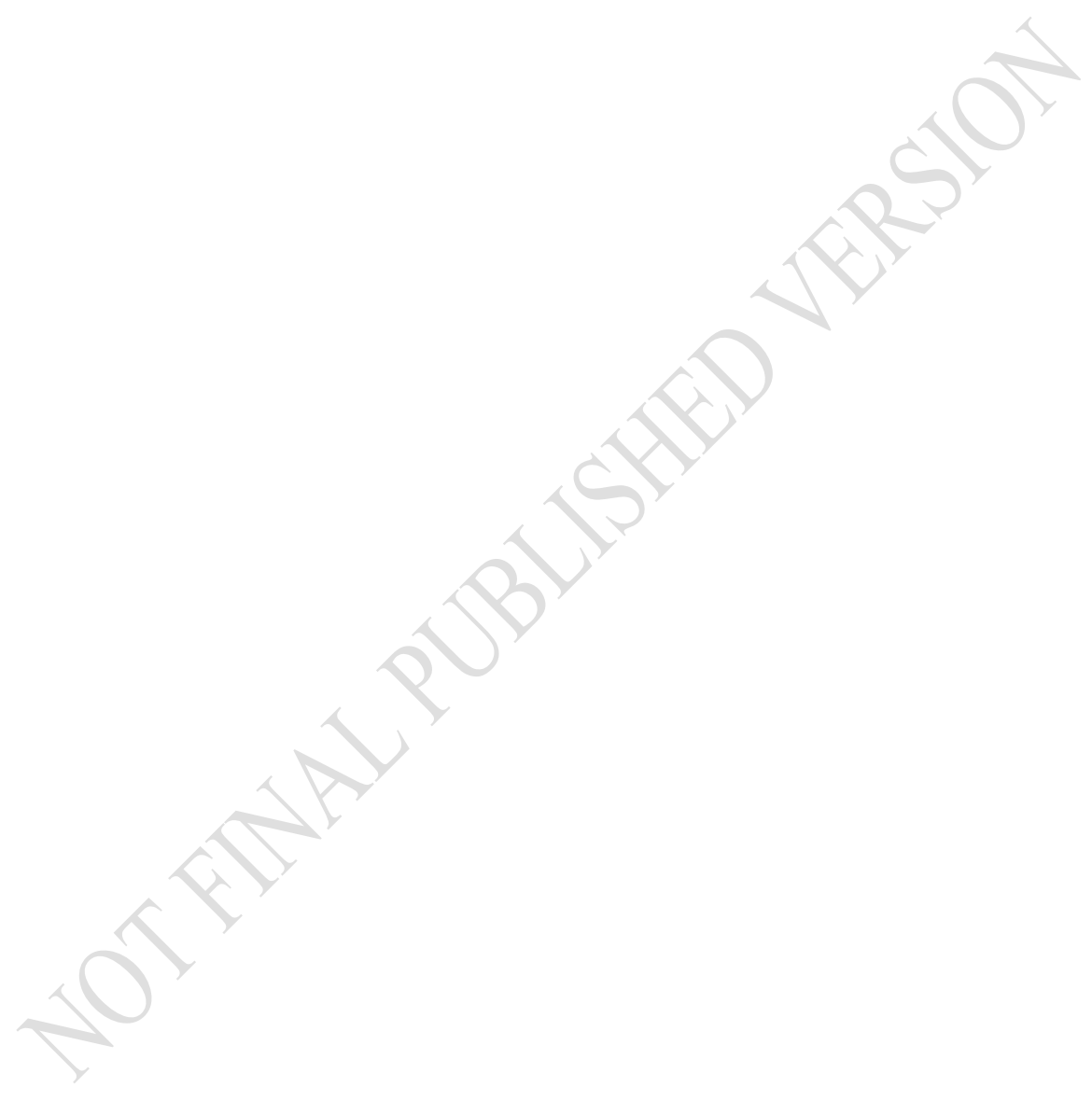

